\title{
Impact of Initial Platelet Count on Baseline Angiographic Finding in Patients with Acute ST- Segment Elevation Myocardial Infarction Undergoing Primary Percutaneous Coronary Intervention
}

\author{
AMIR R. TALHA, M.Sc.; MEDHAT M. ASHMAWY, M.D.; EHAB A. HAMDY, M.D. and \\ ENAS E. DERAZ, M.D.
}

The Department of Cardiology, Faculty of Medicine, Tanta University

\begin{abstract}
Background: Platelets play an important role in cardiovascular disease both in the pathogenesis of atherosclerosis and in the development of acute thrombotic events. Rupture of atheromatous plaque and subsequent occlusive thrombus formation are believed to be responsible for most acute myocardial ischemic events.

Aim of Study: The aim of this study is to assess the relation and effect of initial platelet count on baseline angiographic findings in patients presented with acute ST Segment Elevation Myocardial Infraction (STEMI) undergoing Primary Percutaneous Coronary Intervention (pPCI)

Patients and Methods: This study was conducted at the Department of Cardiovascular Medicine, Tanta University Hospital at the period between June 2017 to December 2017 It was carried out on 150 patients diagnosed definitively with acute STEMI and treated with pPCI. Blood samples were collected from the patient on admission to show initial platelet count before coronary intervention followed by assessment of the angiographic findings before wiring of the infract related artery by TIMI flow grade system and scoring of the coronary anatomy with SYNTAX score.
\end{abstract}

The patients were divided into two groups, group (1): Included patients who had TIMI flow grade 0 ( 82 patients) representing $54.7 \%$ and group (2): Patients who had TIMI flow grade $1-3$ (68 patients) $45.3 \%$.

Results: Group (1) who showed total occlusion of infract related artery (TIMI 0) patients had higher initial platelet count with a mean of $(255.74 \pm 72.01) \times 10^{3} / \mathrm{mm}^{3}$ compared to group (2) (TIMI 1-3) in which patients had lower initial platelet count with a mean of $(194.94 \pm 47.54) \times 10^{3} / \mathrm{mm}^{3}$ There was no significant difference between the two groups as regarding of complexity of coronary arteries anatomy calculated by SYNTAX score.

Conclusion: Initial platelet count in patients presented with acute STEMI had a role in predicting pre-interventional angiographic findings, higher initial platelet count is associated with higher incidence of total occlusion of infract related

Correspondence to: Dr. Amir R. Talha, The Department of Cardiology, Faculty of Medicine, Tanta University artery in STEMI patients treated with primary PCI, there was no significant relation between initial platelet count and SYNTAX score grade, initial platelet count alone has no effect on SYNTAX flow grade of coronary arteries. It seems that it is affected by multiple risk factors.

Key Words: STEMI - Platelets - TIMI - SYNTAX-pPCI.

\section{Introduction}

ISCHAEMIC heart disease is the single most common cause of death [1]. Acute ST-Elevation Myocardial Infarction (STEMI) results from the sudden obstruction of a coronary artery causing abrupt interruption of blood flow to the myocardium as a consequence of intimal rupture or erosion of an atherosclerotic plaque with superimposed thrombosis [2].

The primary goal in management of acute STEMI is reperfusion therapy with intravenous fibrinolysis or pPCI [3]

Primary Percutaneous Coronary Intervention (pPCI) is the preferred reperfusion strategy in patients with STEMI within 12 hours of symptom onset, provided it can be performed by an experienced well trained team. Lower mortality rates among patients undergoing primary PCI are observed in canters with a high volume of PCI procedures [4].

Platelets play an important role in cardiovascular disease both in the pathogenesis of atherosclerosis and in the development of acute thrombotic events. Rupture of atheromatous plaque and subsequent occlusive thrombus formation are believed to be responsible for most acute myocardial ischemic events, such as unstable angina and acute myocardial infarction [2] 


\section{Patients and Methods}

This study was conducted at the Department of Cardiovascular Medicine, Tanta University Hospital at the period between June 2017 \& December 2017. It was carried out on 150 patients diagnosed definitively with acute STEMI and treated with pPCI.

All patients were subjected to detailed history taking, full clinical examination, 12 lead electrocardiogram, echocardiography and primary PCI strategy. In all patients recruited in this study, blood samples were collected on admission before PCI from the ante-cubital vein by an atraumatic puncture and were sent to the laboratory for analysis of: Serum cardiac biomarkers, complete blood count (hemoglobin, platelets, total White Blood Cells (WBCs). All patient received $300 \mathrm{mg}$ acetyl salicylic acid, $600 \mathrm{mg}$ clopidogrel, $80 \mathrm{mg}$ of atorvastatin unfractionated heparin as a loading dose according to the body weight.

All patient underwent pPCI, then preintervention on the infract related artery angiographic findings were assessed by TIMI flow grade system [5]. Then complexity of coronary arteries were scored using the openly accessible web based score calculator (http://www.syntaxscore.com ), SYNTAX score results were categorized as low score (SYNTAX score $\leq 22$ ), intermediate score (SYNTAX score 23-32), and high score (SYNTAX score $\geq 33$ ) [6].

The patients of the study were divided into two groups: Group (1): Included patients who had TIMI flow grade 0 (82 patients) representing $54.7 \%$ and Group (2): Patients who had TIMI flow grade 13 (68 patients) representing $45.3 \%$.

\section{Exclusion criteria:}

Patients on antiplatelets, patients presented with previous STEMI, patients with chronic kidney disease (creatinine clearance $<15 \mathrm{~mL} / \mathrm{min}$ ), patients who previously underwent Coronary Artery Bypass Graft (CABG), patients with hematological disorders, patients with active hepato-biliary disease, patients with active infections, patients with neoplastic diseases and patients with recent major surgical procedure or trauma.

Patients in each group were matched to the other group regarding different demographic, clinical and laboratory parameters.

Duration of the study: This study was done in a period of six months from June 2017 to December 2017.
Statistical analysis: Data were fed to the computer and analyzed using IBM SPSS software package Version 20.0. (Armonk, NY: IBM Corp). Qualitative data were described using number and percent. The Kolmogorov-Smirnov test was used to verify the normality of distribution Quantitative data were described using range (minimum and maximum), mean, standard deviation and median. Significance of the obtained results was judged at the 5\% level. The used tests were:

1- Chi-square test: For categorical variables, to compare between different groups.

2- Fisher's Exact or Monte Carlo correction:For correction of chi-square when more than $20 \%$ of the cells have expected count less than 5 .

3- Student t-test: For normally distributed quantitative variables, to compare between two studied groups.

4- Mann Whitney test: For abnormally distributed quantitative variables, to compare between two studied groups.

5- Kruskal Wallis test: For abnormally distributed quantitative variables, to compare between more than two studied groups.

6- Receiver operating characteristic curve (ROC): It is generated by plotting sensitivity (TP) on Y axis versus specificity (FP) on $\mathrm{X}$ axis at different cut off values. The area under the ROC curve denotes the diagnostic performance of the test. Area more than 50\% gives acceptable performance and area about $100 \%$ is the best performance for the test. The ROC curve allows also a comparison of performance between two tests [7].

\section{Results}

Patient demographics: Regarding the gender: 57 patients of the study population were males (38\%) and 93 were females (62\%). Group I included 29 males (35.4\%) and 53 females (64.6\%), Group 2 included 28 males $(41.2 \%)$ and 40 females $(58.8 \%)$. There was no satistically significant difference between the two groups ( $p$-value $=0.465)$ (Table 1). As regarding age: The age of the study population ranged from 39 to 80 years with a mean of $59.33 \pm 9.98$ years. There was no statistically significant difference between the two groups ( $p$ value $=0.983)($ Table 1$)$.

Prevalence of risk factors: There was no statistically significant differences as regarding cardiovascular risk factors between the two groups except for family history of coronary artery disease ( $p$ value $=0.039$ ) (Table 2). 
Table (1): Comparison between the two studied groups according to demographic data.

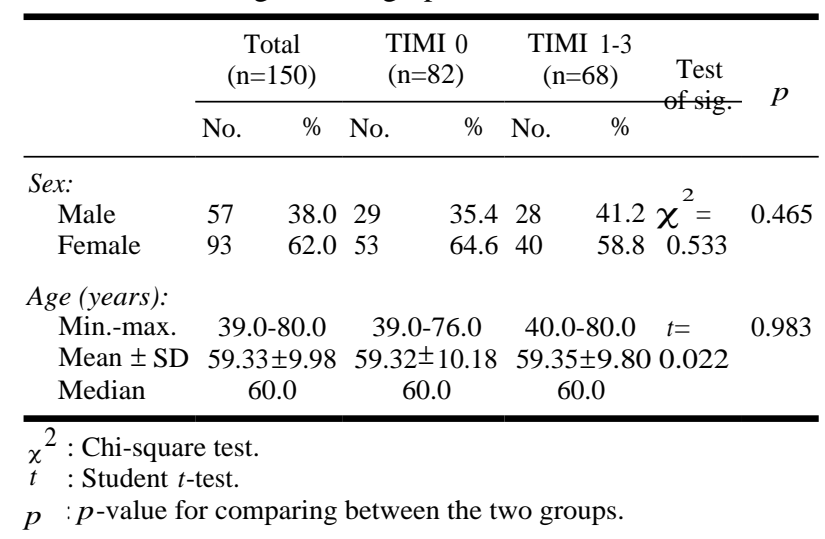

Table (2): Comparison between the two studied groups according to risk factors.

\begin{tabular}{|c|c|c|c|c|c|c|}
\hline & $\begin{array}{c}\text { Total } \\
(\mathrm{n}=150)\end{array}$ & \multirow{2}{*}{$\begin{array}{c}\begin{array}{l}\text { TIMI } 0 \\
(\mathrm{n}=82)\end{array} \\
\text { No. \% }\end{array}$} & \multicolumn{2}{|c|}{$\begin{array}{l}\text { TIMI } 1-3 \\
(n=68)\end{array}$} & \multirow{2}{*}{$x^{2}$} & \multirow{2}{*}{$p$} \\
\hline & No. $\%$ & & No. & $\%$ & & \\
\hline - Diabetic & 9966.0 & 5870.7 & 41 & 60.3 & 1.805 & 0.179 \\
\hline - Hypertension & 8758.0 & 5263.4 & 35 & 51.5 & 2.177 & 0.140 \\
\hline - Dyslipidemic & 7348.7 & 3745.1 & 36 & 52.9 & 0.910 & 0.340 \\
\hline $\begin{array}{l}\text { - Coronary artery } \\
\text { disease. }\end{array}$ & 5536.7 & 3137.8 & 24 & 35.3 & 0.101 & 0.751 \\
\hline $\begin{array}{c}\text { - Family history of } \\
\text { coronary disease }\end{array}$ & 6946.0 & 4453.7 & 25 & 36.8 & $4.271 *$ & $0.039 *$ \\
\hline - Smoking & 8556.7 & 4858.5 & 37 & 54.4 & 0.258 & 0.612 \\
\hline
\end{tabular}

Killip class: 114 patients of the study population presented with killip class I (76.0\%), 29 patients presented with killip class II (19.3\%), 2 patients presented with killip class III $(1.3 \%), 5$ patients presented with killip class IV (3.3\%), there was no statistically significant difference between the studied groups as regarding killip class $(p$-value $=$ 0.099) (Table 3).

Table (3): Comparison between the two studied groups according to Killip class.

\begin{tabular}{llllllllc}
\hline \multirow{2}{*}{$\begin{array}{l}\text { Killip } \\
\text { class }\end{array}$} & \multicolumn{1}{c}{$\begin{array}{c}\text { Total } \\
(\mathrm{n}=150)\end{array}$} & \multicolumn{2}{c}{$\begin{array}{c}\text { TIMI 0 } \\
(\mathrm{n}=82)\end{array}$} & \multicolumn{2}{c}{$\begin{array}{c}\text { TIMI 1-3 } \\
(\mathrm{n}=68)\end{array}$} & & \\
\cline { 2 - 6 } & No. & $\%$ & No. & $\%$ & No. & $\%$ & & $p$ \\
\hline Class I & 114 & 76.0 & 60 & 73.2 & 54 & 79.4 & 5.589 & 0.099 \\
Class II & 29 & 19.3 & 15 & 18.3 & 14 & 20.6 & & \\
Class III & 2 & 1.3 & 2 & 2.4 & 0 & 0.0 & & \\
Class IV & 5 & 3.3 & 5 & 6.1 & 0 & 0.0 & & \\
\hline
\end{tabular}

$\chi^{2}$ : Chi-square test.

$p: p$-value for comparing between the two groups.

STEMI type: 89 patients of the study population were presented with anterior STEMI (59.3\%), 38 patients were presented with inferior STEMI (25.3\%), 5 patients were presented with lateral
STEMI (3.3\%), 7 patients were presented with inferior, right and posterior STEMI (4.7\%), 8 patients were presented with inferior and right STEMI (5.3\%), 3 patients were presented with inferior and posterior STEMI (2\%), there was no statistically significant difference between the studied groups as regarding STEMI type. $(p$-value $=$ 0.987) (Table 4).

Table (4): Comparison between the two studied groups according to type of STEMI.

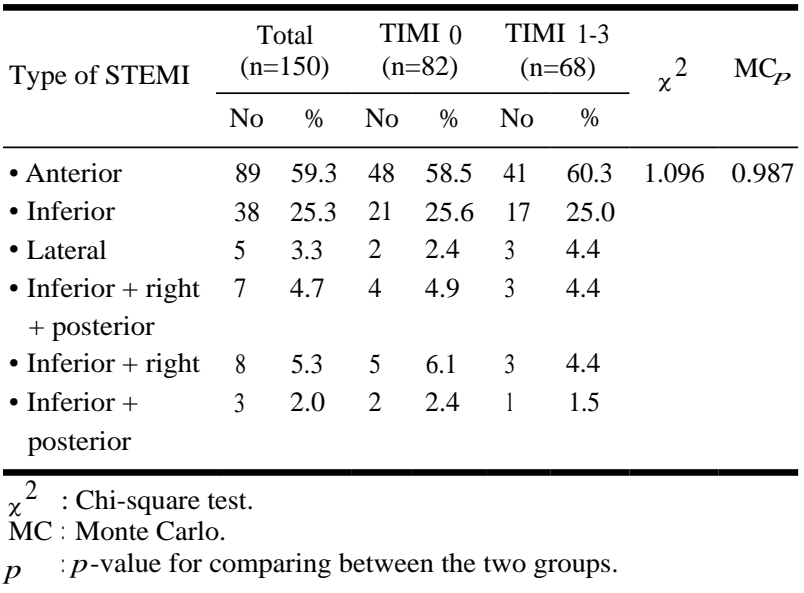

Echocardiographic findings: As regarding ejection fraction\%. In Group 1, ejection fraction\% was (min.-max. $=30.0-62.0$ ) with mean $\pm \mathrm{SD}=46.45 \pm$ 7.17. In Group 2, ejection fraction\% was (min.max. $=32-62.0$ ) with mean $\pm \mathrm{SD}=48.13 \pm 6.87$. There was no statistically significant difference between the studied groups $(p$-value $=0.147)$ (Table $5)$ as regarding resting segmental wall motion abnormalities: RSWMAs were absent in 8 patients of the study population $(5.3 \%), 70$ patients had anterior wall hypokinesia (46.7\%), 52 patients had inferior wall hypokinesia (34.7\%), 3 patients had lateral wall hypokinesia (2\%), 17 patients had RSWMAs in the form of global wall hypokinesia $(11.3 \%)$. There was a statistically significant difference between the studied groups as regarding RSWMAs. $(p$-value $=0.037)($ Table 6)

Table (5): Comparison between the two studied groups according to ejection fraction $\%$.

\begin{tabular}{|c|c|c|c|c|c|}
\hline & $\begin{array}{c}\text { Total } \\
(\mathrm{n}=150)\end{array}$ & $\begin{array}{l}\text { TIMI } 0 \\
(n=82)\end{array}$ & $\begin{array}{c}\text { TIMI } 1-3 \\
(n=68)\end{array}$ & $t$ & $p$ \\
\hline \multicolumn{6}{|l|}{ Ejection } \\
\hline \multicolumn{6}{|l|}{ fraction \%: } \\
\hline Min.-max. & $30.0-62.0$ & $30.0-62.0$ & $32.0-62.0$ & 1.457 & 0.147 \\
\hline Mean \pm SD & $47.21 \pm 7.06$ & $46.45 \pm 7.17$ & $48.13 \pm 6.87$ & & \\
\hline Median & 47.0 & 47.0 & 47.0 & & \\
\hline
\end{tabular}


Table (6): Comparison between the two studied groups according to resting segmental wall motion abnormalities.

\begin{tabular}{|c|c|c|c|c|c|c|c|c|}
\hline \multirow{2}{*}{$\begin{array}{l}\text { Wall motion } \\
\text { abnormalities }\end{array}$} & \multicolumn{2}{|c|}{$\begin{array}{c}\text { Total } \\
(n=150)\end{array}$} & \multicolumn{2}{|c|}{$\begin{array}{l}\text { TIMI } 0 \\
(n=82)\end{array}$} & \multicolumn{2}{|c|}{$\begin{array}{l}\text { TIMI } 1-3 \\
(n=68)\end{array}$} & \multirow{2}{*}{$x^{2}$} & \multirow[t]{2}{*}{$\mathrm{MC}_{p}$} \\
\hline & No. & $\%$ & No. & $\% \mathrm{I}$ & Jo. & $\%$ & & \\
\hline Absent & 8 & 5.3 & 1 & 1.2 & 7 & 10.3 & $9.561^{*}$ & $0.037 *$ \\
\hline Anterior & 70 & 46.7 & 36 & 43.9 & 34 & 50.0 & & \\
\hline Inferior & 52 & 34.7 & 30 & 36.6 & 22 & 32.4 & & \\
\hline Lateral & 3 & 2.0 & 2 & 2.4 & 1 & 1.5 & & \\
\hline Global & 17 & 11.3 & 13 & 15.9 & 4 & 5.9 & & \\
\hline
\end{tabular}

$\chi^{2}$ : Chi-square test.

MC : Monte Carlo.

p $: p$-value for comparing between the two groups.

: Statistically significant at $p \leq 0.05$

\section{Laboratory parameters:}

- Initial platelet count: The initial platelets count of the studięd population ranged from 73.0-562.0 $\left(\mathrm{x}_{10} / \mathrm{mm}_{3}^{3}\right)$ with a mean of $226.82 \pm 69.65$ $\left(\mathrm{x} 10 / \mathrm{mm}_{3}\right)$. In Group 1, it ranged from 73.0$562.0\left(\mathrm{x} 10_{3} / \mathrm{mm}^{3}\right)$ with a mean of $255.74 \pm 72.01$ $\left(\mathrm{x} 10 / \mathrm{mm}^{3}\right)$. In Group 2, it ranged from 90.0$320.0(\mathrm{x} 10 / \mathrm{mm})$ with a mean $194.94 \pm 47.54$ $\left(\mathrm{x} 10 / \mathrm{mm}^{3}\right)$. (Table 7$)$.

There was statistically significant difference between the studied groups as regarding initial platelet count. $(p$-value $=<0.001)$. (Table 7).

Table (7): Comparison between the two studied groụps according to initial platelet count $\left(\times 10 / \mathrm{mm}^{3}\right)$.

\begin{tabular}{llllll}
\hline & $\begin{array}{c}\text { Total } \\
(\mathrm{n}=150)\end{array}$ & $\begin{array}{c}\text { TIMI 0 } \\
(\mathrm{n}=82)\end{array}$ & $\begin{array}{l}\text { TIMI 1-3 } \\
(\mathrm{n}=68)\end{array}$ & $\mathrm{U}$ & $p$ \\
\hline Initial platelet & & & & & \\
count $\left(X_{3}\right.$. & & & & & \\
$\left.10^{3}\right) / \mathrm{mm}^{2}:$ & & & & \\
Min.-max. & $73.0-562.0$ & $73.0-562.0$ & $90.0-320.0$ & $1013.5^{*}$ & $<0.001^{*}$ \\
Mean \pm SD. & $226.82 \pm$ & $255.74 \pm$ & $194.94 \pm$ & \\
& 69.65 & 72.01 & 47.54 & \\
Median & 212.50 & 244.50 & 190.0 & \\
\hline
\end{tabular}

$\mathrm{U}:$ Mann Whitney test.

$p: p$-value for comparing between the two groups.

* : Statistically significant at $p \leq 0.05$.

- Haemoglobin: The haemoglobin level of the studied population ranged from $8.50-16.30 \mathrm{gm} / \mathrm{dL}$ with a mean of $12.51 \pm 1.64 \mathrm{gm} / \mathrm{dL}$. There was no statistically significant difference between the studied groups as regarding haemoglobin level. $(p$-value $=0.513)$. $($ Table 8$)$.

- Total leukocytic count: The total leukocytic count of the studied p̧opulation ranged from $1.40-33.20\left(\times 10^{3} / \mathrm{mm}^{3}\right)$ with a mean of $9.17 \pm$ $4.63\left(\mathrm{x} 10^{3} / \mathrm{mm}^{3}\right)$. There was no statistically significant difference between the studied groups as regarding total leukocytic count. ( $p$-value $=$ 0.802). (Table 8).
Table (8): Comparison between the two studied groups according to hemoglobinn levę $(\mathrm{gm} / \mathrm{dL})$ and total leukocytic count $\left(\times 10 / \mathrm{mm}^{3}\right)$.

\begin{tabular}{|c|c|c|c|c|c|}
\hline & $\begin{array}{c}\text { Total } \\
(\mathrm{n}=150)\end{array}$ & $\begin{array}{l}\text { TIMI } 0 \\
(\mathrm{n}=82)\end{array}$ & $\begin{array}{l}\text { TIMI } 1-3 \\
(n=68)\end{array}$ & $\begin{array}{l}\text { Test } \\
\text { of sig. }\end{array}$ & $p$ \\
\hline \multicolumn{6}{|l|}{$\begin{array}{l}\text { Hemoglobin } \\
\text { level: }\end{array}$} \\
\hline Min.-max. & $8.50-16.30$ & $8.80-16.0$ & $8.50-16.30$ & & 0.513 \\
\hline Mean \pm SD. & $12.51 \pm 1.64$ & $12.59 \pm 1.52$ & $12.42 \pm 1.78$ & 0.655 & \\
\hline Median & 12.25 & 12.40 & 12.05 & & \\
\hline \multicolumn{6}{|l|}{$\begin{array}{l}\text { Total } \\
\text { leukocytic } \\
\text { count }\left(\times 10^{3} /\right. \\
\left.\mathrm{mm}^{3}\right):\end{array}$} \\
\hline Min.-max. & $1.40-33.20$ & $1.90-33.20$ & $1.40-22.0$ & $\mathrm{U}=$ & 0.802 \\
\hline Mean \pm SD & $9.17 \pm 4.63$ & $9.42 \pm 5.16$ & $8.87 \pm 3.91$ & 2721.5 & \\
\hline Median & 8.50 & 9.55 & 8.20 & & \\
\hline
\end{tabular}

\section{Angiographic findings:}

- Culprit vessel: In 89 patients of the study population the Left Anterior Descending artery (LAD) was the culprit vessel $(59.3 \%)$, while in 45 patients it was the Right Coronary Artery (RCA) $(30 \%)$, and in 11 patients the culprit vessel was the left circumflex artery (LCX) $(7.3 \%)$, while in 3 patients it was the first diagonal branch (D1) $(2 \%)$, in 1 patient it was the second diagonal branch (D2) $(0.7 \%)$, and 1 patient it was the ramus branch $(0.7 \%)$. There was no statistically significant difference between the studied groups as regarding the culprit vessel. $(p$-value $=0.393)$ (Table 9).

Table (9): Comparison between the two studied groups according to culprit vessel.

\begin{tabular}{|c|c|c|c|c|c|c|c|c|}
\hline \multirow{2}{*}{$\begin{array}{l}\text { Culprit } \\
\text { vessel }\end{array}$} & \multicolumn{2}{|c|}{$\begin{array}{c}\text { Total } \\
(\mathrm{n}=150)\end{array}$} & \multicolumn{2}{|c|}{$\begin{array}{l}\text { TIMI } 0 \\
(\mathrm{n}=82)\end{array}$} & \multicolumn{2}{|c|}{$\begin{array}{l}\text { TIMI } 1-3 \\
(\mathrm{n}=68)\end{array}$} & \multirow[t]{2}{*}{$x^{2}$} & \multirow[t]{2}{*}{$\mathrm{MC}_{p}$} \\
\hline & No. & $0 / 0$ & No. & $\%$ & No. & $\%$ & & \\
\hline LAD & 89 & 59.3 & 48 & 58.5 & 41 & 30.3 & 4.978 & 0.393 \\
\hline $\mathrm{RCA}$ & 45 & 30.0 & 26 & 31.7 & 19 & 27.9 & & \\
\hline LCX & 11 & 7.3 & 6 & 7.3 & 5 & 7.4 & & \\
\hline D1 & 3 & 2.0 & 0 & 0.0 & 3 & 4.4 & & \\
\hline D2 & 1 & 0.7 & 1 & 1.2 & 0 & 0.0 & & \\
\hline RAMUS & 1 & 0.7 & 1 & 1.2 & 0 & 0.0 & & \\
\hline
\end{tabular}

- Number of diseased vessels: 54 patients of the study population had a single vessel disease (36\%), while 42 patients had two vessels disease $(28 \%)$ and 54 patients had three vessels disease $(36 \%)$. There was no statistically significant difference between the studied groups as regarding the number of the diseased vessel. ( $p$-value $=$ 0.400) (Table 10). 
Table (10): Comparison between the two studied groups according to number of diseased vessels.

\begin{tabular}{|c|c|c|c|c|c|c|c|c|}
\hline \multirow{2}{*}{$\begin{array}{l}\text { Number of } \\
\text { diseased } \\
\text { vessels }\end{array}$} & \multicolumn{2}{|c|}{$\begin{array}{c}\text { Total } \\
(\mathrm{n}=150)\end{array}$} & \multicolumn{2}{|c|}{$\begin{array}{l}\text { TIMI } 0 \\
(\mathrm{n}=82)\end{array}$} & \multicolumn{2}{|c|}{$\begin{array}{l}\text { TIMI 1-3 } \\
(\mathrm{n}=68)\end{array}$} & \multirow{2}{*}{$\begin{array}{c}\text { Test } \\
\text { of sig. }\end{array}$} & \multirow{2}{*}{$p$} \\
\hline & No. & $\%$ & No. & $\%$ & No. & $\%$ & & \\
\hline 1 & 54 & 36.0 & 26 & 31.7 & 28 & 41.2 & $\chi^{2}=$ & 0.400 \\
\hline 2 & 42 & 28.0 & 26 & 31.7 & 16 & 23.5 & 1.831 & \\
\hline 3 & 54 & 36.0 & 30 & 36.6 & 24 & 35.3 & & \\
\hline Min.-max. & \multicolumn{2}{|c|}{$1.0-3.0$} & \multicolumn{2}{|c|}{$1.0-3.0$} & \multicolumn{2}{|c|}{$1.0-3.0$} & $\mathrm{U}=$ & 0.441 \\
\hline Mean \pm SD. & \multicolumn{2}{|c|}{$2.0 \pm 0.85$} & \multicolumn{2}{|c|}{$2.05 \pm 0.83$} & \multicolumn{2}{|c|}{$1.94 \pm 0.88$} & 2596.0 & \\
\hline Median & \multicolumn{2}{|c|}{2.0} & \multicolumn{2}{|c|}{2.0} & \multicolumn{2}{|c|}{2.0} & & \\
\hline
\end{tabular}

$\chi^{2}:$ Chi-square test.

U : Mann Whitney test.

$p: p$-value for comparing between the two groups.

- Number of deployed stents during the procedure: 2 stents were deployed in 19 patients of the study population $(12.7 \%)$, while in 113 patients 1 stent was deployed (75.3\%), deferred stenting in 18 patients (12\%). In Group 1, 2 stents were deployed in 16 patients $(19.5 \%)$, while in 52 patients 1 stent was deployed (63.4\%), deferred stenting in 14 patients $(17.1 \%)$. In Group 2, 2 stents were deployed in 3 patients $(4.4 \%)$, while in 61 patients 1 stent was deployed (89.7\%), deferred stenting in 4 patients $(5.9 \%)$. There was statistically significant difference between the studied groups as regarding the number of deployed stents. ( $p$ value $=0.001)($ Table 11)

Table (11): Comparison between the two studied groups according to number of deployed stents.

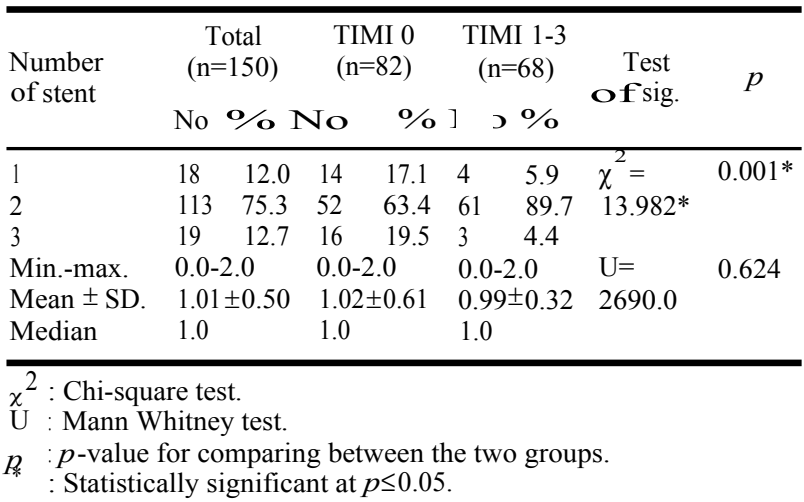

- Usage of ballon dilatation during the procedure: In 100 patients of the study population ballon dilatation was used during intervention $(66.7 \%)$. In Group 1, in 61 patients ballon dilatation was used (74.4\%). In Group 2, ballon dilatation was used in 39 patients $(57.4 \%)$. There was statistically significant difference between the studied groups as regarding intervention with ballon dilatation during the procedure. $(p$-value $=0.028)$ (Table 12).

- Intervention by direct stenting: In 132 patients of the study population intervention was by direct stenting in the infract related artery $(88 \%)$. In
Group 1, 68 patients direct stenting was done $(82.9 \%)$ while In Group 2, direct stenting was in 64 patients $(94.1 \%)$. There was statistically significant difference between the studied groups as regarding intervention by direct stenting. ( $p$ value $=0.036)$ (Table 12).

- Deferred stenting and intervention by only ballon dilatation: 18 patients of the study population had intervention with only ballon dilatation with deferred stenting (12\%). In Group 1, they were 14 patients (17.1\%). In Group 2, they were 4 patients $(5.9 \%)$. There was statistically significant difference between the studied groups as regarding deferred stenting and intervention by only ballon dilatation. $(p$-value $=0.036)($ Table 12) .

- Coronary artery dissection: In 3 patients of the study population coronary intervention was complicated by dissection $(2 \%)$. There was no statistically significant difference between the studied groups as regarding development of coronary artery dissection. $(p$-value $=1.000)$ (Table 12).

- Successful intervention: In 145 patients of the study population coronary intervention was successful $(96.7 \%)$. There was no statistically significant difference between the studied groups as regarding successful intervention. ( $p$-value $=$ 0.064) (Table 12).

Table (12): Comparison between the two studied groups according to different parameters.

\begin{tabular}{|c|c|c|c|c|c|c|c|c|}
\hline & \multicolumn{2}{|c|}{$\begin{array}{c}\text { Total } \\
(n=150)\end{array}$} & \multicolumn{2}{|c|}{$\begin{array}{l}\text { TIMI } 0 \\
(n=82)\end{array}$} & \multicolumn{2}{|c|}{$\begin{array}{l}\text { TIMI 1-3 } \\
(n=68)\end{array}$} & \multirow{2}{*}{$x^{2}$} & \multirow{2}{*}{$p$} \\
\hline & No. & $\%$ & No. & $\%$ & No. & $\%$ & & \\
\hline $\begin{array}{l}\text { - Intervention } \\
\text { by ballon } \\
\text { dilatation. }\end{array}$ & 100 & 66.7 & 61 & 74.4 & 39 & 57.4 & $4.856^{*}$ & $0.028 *$ \\
\hline $\begin{array}{l}\text { - Intervention } \\
\text { with direct } \\
\text { stenting. }\end{array}$ & 132 & 88.0 & 68 & 82.9 & 64 & 94.1 & $4.409^{*}$ & $0.036^{*}$ \\
\hline $\begin{array}{l}\text { Deferred } \\
\text { stenting. }\end{array}$ & 18 & 12.0 & 14 & 17.1 & 4 & 5.9 & $4.409^{*}$ & $0.036^{*}$ \\
\hline $\begin{array}{l}\text { Ballon } \\
\text { dilatation } \\
\text { only. }\end{array}$ & 18 & 12.0 & 14 & 17.1 & 4 & 5.9 & $4.409^{*}$ & $0.036^{*}$ \\
\hline $\begin{array}{l}\text { - Successful } \\
\text { intervention. }\end{array}$ & 145 & 96.7 & 77 & 93.9 & 68 & 100.0 & 4.289 & $\begin{array}{l}\mathrm{FE}_{p=} \\
0.064\end{array}$ \\
\hline • Dissection. & 3 & 2.0 & 2 & 2.4 & 1 & 1.5 & 0.178 & $\begin{array}{l}\mathrm{FE}_{p=}= \\
1.000\end{array}$ \\
\hline
\end{tabular}

$\chi^{2}:$ Chi square test

FE : Fisher Exact.

$p_{*}: p$-value for comparing between the two groups.

: Statistically significant at $p \leq 0.05$.

- Syntax score grade: 124 patients of the study population had low syntax score grade $(82.7 \%)$, 25 patients had intermediate syntax score grade $(16.7 \%)$, while 1 patient had high syntax score grade $(0.7 \%)$. In Group 1,65 patients had low 
syntax score grade $(79.3 \%), 16$ patients had intermediate syntax score grade $(19.5 \%)$, while 1 patient had high syntax score grade $(1.2 \%)$. In Group 2, 59 patients had low syntax score grade (86.8\%), 9 patients had intermediate syntax score grade $(13.2 \%)$. There was no statistically significant difference between the studied groups as regarding syntax score grade. $(p$-value $=0.386)$ (Table 13).

Table (13): Comparison between the two studied groups according to Syntax score grade.

\begin{tabular}{|c|c|c|c|c|c|c|c|c|}
\hline \multirow{2}{*}{$\begin{array}{l}\text { Syntax } \\
\text { score grade }\end{array}$} & \multicolumn{2}{|c|}{$\begin{array}{c}\text { Total } \\
(n=150)\end{array}$} & \multicolumn{2}{|c|}{$\begin{array}{l}\text { TIMI } 0 \\
(n=82)\end{array}$} & \multicolumn{2}{|c|}{$\begin{array}{l}\text { TIMI } 1-3 \\
(n=68)\end{array}$} & \multirow{2}{*}{$x^{2}$} & \multirow[t]{2}{*}{$\mathrm{MC}_{p}$} \\
\hline & No. & $\% I$ & Jo. & $\% I$ & Jo. & $\%$ & & \\
\hline Low & 124 & 82.7 & 65 & 79.3 & 59 & 86.8 & 1.868 & 0.386 \\
\hline Intermediate & 25 & 16.7 & 16 & 19.5 & 9 & 13.2 & & \\
\hline High & 1 & 0.7 & 1 & 1.2 & 0 & 0.0 & & \\
\hline
\end{tabular}

- TIMI flow grade post coronary intervention: 5 patients of the study population had TIMI 0 (3.3\%), 5 patients had TIMI 1 (3.3\%), 18 patients had TIMI 2 (12\%), while 122 patients had TIMI $3(81.3 \%)$. In Group 1, 5 patients had TIMI 0 (6.1\%), 4 patients had TIMI 1 (4.9\%), 15 patients had TIMI $2(18.3 \%)$, while 58 patients had TIMI $3(70.7 \%)$. In Group 2, 1 patient had TIMI 1 (1.5\%), 3 patients had TIMI $2(4.4 \%)$, while 64 patients had TIMI 3 (94.1\%). There was statistically significant difference between the studied groups as regarding TIMI flow grade post intervention. $(p$-value $=0.001)($ Table 14) .

Table (14): Comparison between the two studied groups according to TIMI flow post intervention.

\begin{tabular}{|c|c|c|c|c|c|c|c|c|}
\hline \multirow{2}{*}{$\begin{array}{l}\text { TIMI flow } \\
\text { post } \\
\text { intervention }\end{array}$} & \multicolumn{2}{|c|}{$\begin{array}{c}\text { Total } \\
(n=150)\end{array}$} & \multicolumn{2}{|c|}{$\begin{array}{l}\text { TIMI } 0 \\
(n=82)\end{array}$} & \multicolumn{2}{|c|}{$\begin{array}{l}\text { TIMI } 1-3 \\
(\mathrm{n}=68)\end{array}$} & \multirow[t]{2}{*}{$x^{2}$} & \multirow[t]{2}{*}{$\mathrm{MC}_{p}$} \\
\hline & No. & $\%$ & No. & $\%$ & No. & $\%$ & & \\
\hline TIMI 0 & 5 & 3.3 & 5 & 6.1 & 0 & 0.0 & \multirow[t]{4}{*}{$13.744 *$} & \multirow[t]{4}{*}{$0.001 *$} \\
\hline TIMI 1 & 5 & 3.36 & 4 & 4.9 & 1 & 1.5 & & \\
\hline TIMI 2 & 18 & 12.0 & 15 & 18.3 & 3 & 4.4 & & \\
\hline TIMI 3 & 122 & 81.3 & 58 & 70.7 & 64 & 94.1 & & \\
\hline \multicolumn{9}{|c|}{$\begin{array}{l}\chi^{2}: \text { Chi-square test. } \\
\text { MC: Monte Carlo. }\end{array}$} \\
\hline $\begin{array}{ll}p_{*} & : p \text {-valu } \\
: \text { Statist }\end{array}$ & $\begin{array}{l}\text { for } \mathrm{c} \\
\text { ally }\end{array}$ & $\begin{array}{l}\text { mpari } \\
\text { onific }\end{array}$ & $\mathrm{g}$ be & $\begin{array}{l}\text { ween } \\
p \leq 0.0\end{array}$ & & & & \\
\hline
\end{tabular}

\section{- Relation between Syntax grade and Initial platelet} count in each group:

- In Group 1 (TIMI 0) with initial platelet count mean $\pm \mathrm{SD}=255.74 \pm 72.01\left(\mathrm{X} \mathrm{10}^{3} / \mathrm{mm}^{3}\right)$ : Patients with high Syntax flow grade was 1 patient with initial platelet count $73000 / \mathrm{mm}^{3}$, patients with intermediate Syntax score grade who were 16 patients had initial platelet count mean $\pm \mathrm{SD}=$
$244.94 \pm 56.95\left(\mathrm{X} 10^{3} / \mathrm{mm}^{3}\right)$, while patients with low Syntax score grade (65 patients) had initial platelet count mean $\pm \mathrm{SD}=261.22 \pm 72.26\left(\times 10^{3}\right.$ / $\mathrm{mm}^{3}$ ). There was no statistically significant difference between the initial platelet count and Syntax score grade. $(p$-value $=0.148)$. $($ Table 15$)$.

- In Group 2 (TIMI 1:3) with initial platelet

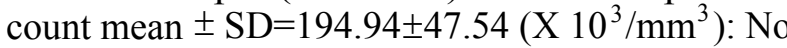
one was with high Syntax flow, patients with intermediate syntax score grade who were 9 patients had initial platelet count mean $\pm \mathrm{SD}=177.33 \pm 65.55$ $\left(\mathrm{X} 10^{3} / \mathrm{mm}^{3}\right)$, while patients with low Syntax score grade (59 patients) had initial platelet count mean $\pm \mathrm{SD}=194.17 \pm 44.49\left(\mathrm{X} \mathrm{10}^{3} / \mathrm{mm}^{3}\right)$. There was no statistically significant difference between the initial platelet count and Syntax score grade. ( $p$ value $=0.328)$. (Table 15).

Table (15): Relation bętween Syntax grade and initial platelet count $\left(\times 10 \% \mathrm{~mm}^{3}\right)$ in each group.

\begin{tabular}{|c|c|c|c|c|c|}
\hline \multirow{2}{*}{$\begin{array}{l}\text { Initial } \\
\text { platelet count } \\
\left(\times 10^{3}\right) / \mathrm{mm}^{-}\end{array}$} & \multicolumn{3}{|c|}{ Syntax grade } & \multirow{2}{*}{$\begin{array}{c}\text { Test } \\
\text { of sig. }\end{array}$} & \multirow{2}{*}{$p$} \\
\hline & Low & Intermediate & High & & \\
\hline Total $(n=150)$ : & $(n=124)$ & $(n=25)$ & $(n=1)$ & & \\
\hline Min.-max. & $90.0-562.0$ & $90.0-370.0$ & 73.0 & $\mathrm{H}=$ & 0.211 \\
\hline Mean \pm SD & $229.31 \pm 69.14$ & $220.60 \pm 67.50$ & & 3.155 & \\
\hline Median & 214.50 & 211.0 & & & \\
\hline TIMI $0(n=82)$ : & $(n=65)$ & $(n=16)$ & $(n=1)$ & & \\
\hline Min.-max. & $90.0-562.0$ & $165.0-370.0$ & 73.0 & $\mathrm{H}=$ & 0.148 \\
\hline Mean \pm SD & $261.22 \pm 72.26$ & $244.94 \pm 56.95$ & & 3.823 & \\
\hline Median & 250.0 & 230.50 & & & \\
\hline TIMI 1-3 $(n=68)$ : & $(n=59)$ & $(n=9)$ & $(n=0)$ & & \\
\hline Min.-max. & $108-320.0$ & $90.0-313.0$ & & $\mathrm{U}=$ & 0.328 \\
\hline Mean \pm SD & $194.17 \pm 44.49$ & $177.33 \pm 65.55$ & & 211.5 & \\
\hline Median & 190.0 & 190.0 & & & \\
\hline
\end{tabular}

- Periprocedural death: 4 patients died during the procedure (2.7\%). In Group 1, they were 3 patients (3.7\%), while in Group 2, 1 patient died during the procedure $(1.5 \%)$. There was no statistically significance between the studied groups. ( $p$-value $=0.627)$. $($ Table 16).

Table (16): Comparison between the two studied groups according to periprocedural death.

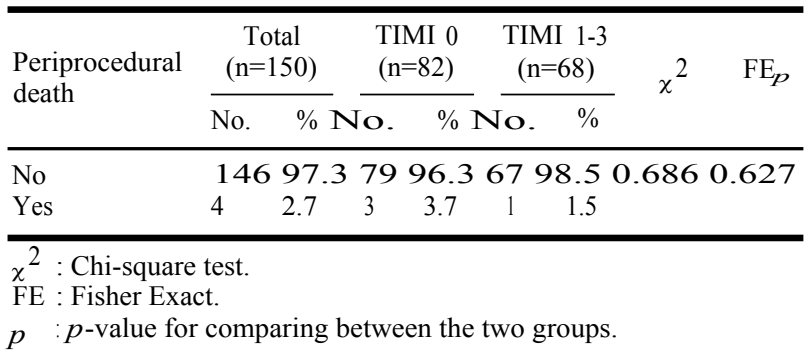


The Receiver Operating Characteristic (ROC) analysis showing the performance and predictive accuracy of initial platelet count in predicting TIMI flow grade 0 . The Area Under the Curve (AUC) was $0.818(p<0.001)_{s}$ with initial platelet count more than $>212(\mathrm{x} 10 / \mathrm{mm})$, with $78.05 \%$ sensitivity and $83.82 \%$ specificity. (Table 17) Fig. (1).

Table (17): Agreement (sensiţivity, specificity) initial platelet count $\left(\times 10^{3}\right) / \mathrm{mm}^{3}$ to predict TIMI 0 cases.

\begin{tabular}{|c|c|c|c|c|}
\hline & Cut off & Sensitiv & pecific & PPV NPV \\
\hline $\begin{array}{l}\text { Initiąl platelet count } \\
\left(\times 10^{3}\right) / \mathrm{mm}\end{array}$ & $>211$ & 78.05 & 83.82 & 85.376 .0 \\
\hline
\end{tabular}

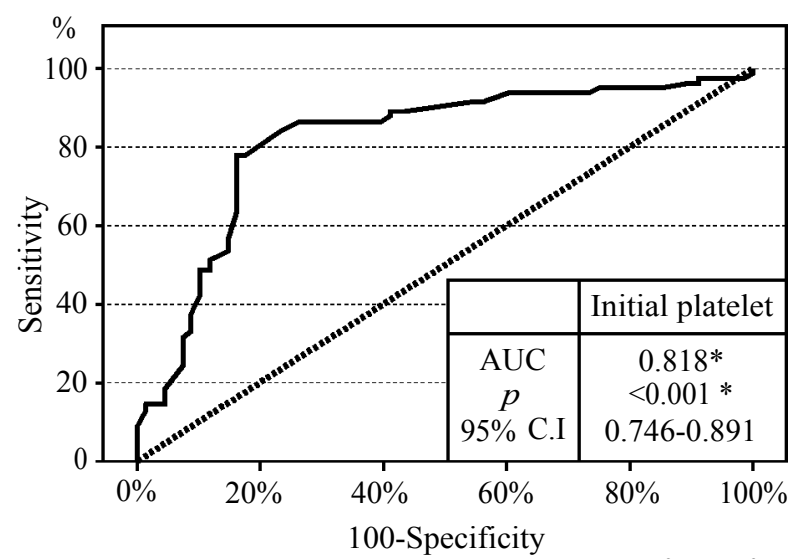

Fig. (1): ROC curve for Initial platelet count $\left(\times 10^{3}\right) / \mathrm{mm}^{3}$ to predict TIMI 0 cases.

\section{Discussion}

ST-Elevation Myocardial Infarction (STEMI) is one of the leading causes of mortality and morbidity worldwide. However, survival after acute STEMI has considerably improved due to increasing symptom recognition, accurate diagnosis and effective timely reperfusion [8]. Reduction in STEMI mortality can be explained by greater use of Percutaneous Coronary Intervention (PCI), antithrombotic therapy and secondary cardiovascular prevention strategies [9]. Platelets play an important role in cardiovascular disease both in the pathogenesis of atherosclerosis and in the development of acute thrombotic events.

Coronary artery thrombosis is the final pathogenic mechanism of acute ischemic events, including myocardial infarction and sudden cardiac arrest.

In our study our aim is trying to show the effect of initial platelet count on baseline angiographic findings assessed with TIMI flow in STEMI patients undergoing primary PCI, in our study which was conducted on 150 patients presented with acute ST segment elevation myocardial infraction then underwent primary PCI. The study sample was divided into two groups: Group 1: Included patients who had TIMI flow grade 0 (82 patients). Group 2: Included patients who had TIMI flow grade 13 (68 patients), patients in each group were matched to the other group regarding different demographic, clinical and laboratory parameters.

As regard age and sex in our study there was no significant difference between the two groups as regarding age $(p$-value $=0.983)$ and sex $(p$-value $=$ 0.465 ). In our study most of the patients who presented with acute STEMI were females (93 patients) $62 \%$.

As regarding 2017 ESC guidelines ischemic heart disease develops on average 7-10 years later in women compared with men. MI remains a leading cause of death in women. Acute Coronary Syndrome (ACS) occurs three to four times more often in men than in women below the age of 60 years, but after the age of 75 , women represent the majority of patients [10]. Women tend to present more often with atypical symptoms, up to $30 \%$ in some registries [11] and tend to present later than men [12]. In the study conducted by Sharif et al., [13] there was no significant difference between age and sex in relation to TIMI flow grade in patients presented with STEMI undergoing $\mathrm{pPC}$.

As regarding diabetes mellitus and systemic hypertension there were no statistically significant difference between the study groups as regarding those parameters in our study. Similarly, the study conducted by Sahin et al., [14] showed no significant difference between diabetes mellitus and systemic hypertension with TIMI flow grade in STEMI patients undergoing primary PCI.

As regarding dyslipidemia and history of coronary artery disease our study could not find significant difference between those parameters and TIMI flow grade. Similarly in the study conducted by Halit et al., [15] which was conducted on 324 patients presented with STEMI and aimed to assess novel predictors of infarct-related artery patency for STEMI that showed no significant difference between dyslipidemia, history of coronary artery diseases and pre-procedural infract related artery patency in patients presented with STEMI undergoing primary PCI. In contrast, a prospective study was conducted by Durmuş et al., [16] on 880 patients with STEMI patients undergoing primary PCI to predict pre-interventional coronary artery patency which showed a significant difference between 
dyslipidemia and infract related coronary artery patency $(p$-value $=0.049)$.

As regarding family history of coronary artery disease in our study there was a significant difference between the study groups. In Group 1 (TIMI $0), 44$ patients had positive family history $(53.7 \%)$, while in Group 2 (TIMI 1-3), 25 patients had family history of coronary artery disease $(36.8 \%)$ ( $p$-value $=0.039$ ). In contrast the study conducted by Durmщ et al., [16] on 880 patients with STEMI patients undergoing primary PCI to predict pre-interventional coronary artery patency which showed no significant difference between family history of coronary artery disease and infract related coronary artery patency.

As regarding smoking in our study there was no significant difference between the study groups and smoking. Similarly in the study conducted by Mathieu et al., [17] on 140 patients presented with STEMI undergoing primary PCI to assess effect of TIMI coronary flow in the culprit coronary artery on myocardial infarct and Microvascular Obstruction (MVO) size. There was no significant difference between smoking and pre-interventional TIMI flow.

As regarding KILLIP class: In our study there was no significant difference between the studied groups as regarding KILLIP class. In contrast a retrospective study was conducted by Halit et al., [15] on 324 patients to assess predictors of infarctrelated artery patency for ST-segment elevation myocardial infarction showed significant difference between the study groups as regarding KILLIP class $(p$-value $=0.031)$.

As regarding initial platelet count, in our study we found that Group 1 that showed total occlusion of infract related artery (TIMI 0 ) has higher initial platelet count with a mean of $(255.74 \pm 72.01)$ $\mathrm{x} 10^{3} / \mathrm{mm}^{3}$ compared to Group 2 (TIMI 1-3) that had lower initial platelet count with a mean of $(194.94 \pm 47.54) \times 10 \% \mathrm{~mm}$. There was statistically significant difference between the studied groups as regarding initial platelet count. ( $p$-value $<0.001$ ).

A study was conducted to assess effect of platelet count on predicting angiographic finding in STEMI patient who underwent primary PCI by Sahin et al., [14]. The study was done on 140 patients and showed that patients with TIMI flow grade 0 had iniţial platelet count with mean of $293.7 \pm 59.8 \times 10^{3} / \mathrm{mm}^{3}$ which was higher than in patients who had higher TIMI flow grade (TIMI $1-3$ ) whoşe mean platelet count was $237.7 \pm 50.9$ $\mathrm{x} 10 \% \mathrm{~mm}^{3}(p$-value $=<0.0001)$.
Similarly in 2016 Sharif et al., [13] in their study to test the effect of platelet count on admission in patients with acute STEMI treated with pPCI on coronary flow, noted that patients who had low platelet count on admission had higher prevalence of TIMI flow grade 3, while patients with high platelet counts on admission has higher incidence of lower TIMI flow grade with more thrombotic infract related artery $(p$-value $=<0.05)$.

A retrospective study was conducted by Halit et al., [15] on 324 patients to predict the patency of the infract related artery in acute myocardial infraction observed that patients who had high TIMI flow grade (TIMI 3) had low mean platelet count with $(248.8 \pm 68.3) \times 10^{3} / \mathrm{mm}^{3}$ as compared to patients who had TIMI flow grade $0,1,2$ whose platelet count was higher with a mean of $252.7 \pm$ $63.4 \times 10^{3} / \mathrm{mm}^{3}$, but without significant difference.

In contrast, a study was conducted by Rui Wang et al., [18] to determine risk factors affecting the in-hospital prognosis of patients with acute ST segment elevation myocardial infarction after PCI and did not conclude that platelet count was an independent risk factor in severity of angiographic findings and in prognosis of coronary artery disease.

Retrospective study was conducted by M Bahrehmand et al., [19] to predict factors affecting thrombolysis in myocardial infraction (TIMI flow) in patients presented with acute STMI which showed that there was no significant difference between initial platelet and pre-interventional angiographic findings assessed with TIMI flow.

As regarding haemoglobin level, we could not find a significant difference between the studied groups as regarding haemoglobin level.

As regarding white blood cells count, we could not find a significant difference between the studied groups as regarding white blood cells count. Similarly a retrospective study was conducted by Halit et al., [15] on 324 patients to test novel predictors of infarct-related artery patency in STEMI demonstrated that no significant difference between white blood cells count and angiographic findings assessed with TIMI flow.

As regarding RSWMAs assessed by echocardiography in our study there was significant difference between the studied groups as regarding RSWMAs. In Group 1 (TIMI 0) 81 patients (98.8\%) had RSWMAs while in Group 2 (TIMI 1-3) 61 patients $(89.7 \%$ ) had RSWMAs ( $p$-value $=0.037$ ), as noticed most of the patients had RSWMAs similarly the study that was conducted by Sabia $\mathrm{p}$ 
et al., [20] noted that most of patient admitted with acute STEMI had RSWMAs ( $p$-value $=>0.001)$.

As regarding Number of stents deployed during the procedure in our study there was significant difference between the studied groups as regarding number of stents used during the procedure. The usage of more than one stent was more in patients with TIMI 0 than in those with TIMI 1-3 ( $p$-value= $0.001)$.

As regarding usage of ballon dilatation during the procedure, in our study there was significant difference between the studied groups as regarding usage of ballon dilatation during the procedure. The usage of ballon dilatation was more in patients with TIMI 0 than in those with TIMI 1-3. ( $p$-value= 0.028).

As most of patients with total occlusion of infract related artery had high thrombotic burden with more distal lesions that appeared after wiring of the site of coronary occlusion usage of more than one stent and ballon dilatation was higher in patients with TIMI flow 0 than in patients with TIMI 1-3.

As regarding direct stenting without ballon dilatation, in our study there was significant difference between the studied groups. Direct stenting was more was more in patients with TIMI 1-3 (Group 2) than in those with TIMI 0 (Group 1) ( $p$ value $=0.036$ ). This was due to less thrombotic field detected in patients with TIMI 1-3.

As regarding deferred stenting during the procedure and only intervention with ballon dilatation, in our study there was significant difference between the studied groups, it was done because of high thrombotic burden and the possibility of immediate stent thrombosis, in Group 1 (TIMI 0), deferred stenting was in 14 patients $(17.1 \%)$. In Group 2 (TIMI 1:3), they were 4 patients $(5.9 \%)$. $(p$-value $=0.036)$.

As regarding TIMI flow grade post coronary intervention in our study there was signific ant difference between the studied groups that showed that prevalence of TIMI 3 was more in Group 2. In Group (1), 58 patients had TIMI 3 (70.7\%), while in Group (2), 64 patients had TIMI 3 (94.1\%). $(p$-value $=0.001)$. In contrast, a study conducted by Schaaf et al., [17] to assess pre-PCI angiographic TIMI flow in the culprit coronary artery influences infarct size and microvascular obstruction in STEMI patients showed no significant difference between pre-interventional and post-interventional TIMI flow.
As regarding the complexity of coronary arteries lesions which were assessed by Syntax score in our study there was no significant statistical difference between initial platelet count and complexity of coronary artery disease. In our study there was no significant difference between TIMI flow grade and complexity of coronary artery lesions assessed by syntax score.

In contrast to our study a study was conducted by Alparslan Kurtul et al., [21] to evaluate the association of platelet to lymphocyte ratio with severity and complexity of coronary artery disease in patients with acute coronary syndromes that noted that patients with high Syntax score had higher mean platelet count than patients with low Syntax score $(p$-value $=>0.001)$.

Limitations of the study: The study had some limitations. This is a single-center experience and represents a limited number of patients.

Many patients presented with acute STEMI have been excluded from the study because they had previous STEMI and others in whom the reperfusion strategy was fibrinolytic therapy.

\section{Conclusion:}

Initial platelet count in patients presented with acute STEMI had a rule in predicting preinterventional angiographic findings, higher initial platelet count is associated with higher incidence of total occlusion of infract related artery in STEMI patients treated with primary PCI.

There is no significant relation between initial platelet count and SYNTAX score grade, initial platelet count alone has no effect on SYNTAX flow grade of coronary arteries, it seems that it is affected by multiple risk factors.

\section{References}

1- HARTLEY A., MARSHALL D.C., SALCICCIOLI J.D., SIKKEL M.B., MARUTHAPPU M. and SHALHOUB J.: Trends in mortality from ischemic heart disease and cerebrovascular disease in Europe: 1980 to 2009. Circulation, 133 (20), 2016.

2- DAVIES M.J. and THOMAS A.: Thrombosis and Acute Coronary-Artery Lesions in Sudden Cardiac Ischemic Death. N. Engl. J. Med., 310 (18): 1137-40, 1984.

3- Correction to: 2016 ACC/AHA Guideline Focused Update on Duration of Dual Antiplatelet Therapy in Patients With Coronary Artery Disease: A Report of the American College of Cardiology/American Heart Association Task Force on Clinical Practice Guidelines: A. Circulation, Sep., 134 (10): e192-4, 2016.

4- THIEMANN D.R., CORESH J., OETGEN W.J. and POWE N.R.: The association between hospital volume 
and survival after acute myocardial infarction in elderly patients. N. Engl. J. Med., 340 (21): 1640-8, 1999.

5- GIBSON C.M., CANNON C.P., MURPHY S.A., MARBLE S.J., BARRON H.V., BRAUNWALD E., et al.: Relationship of the TIMI myocardial perfusion grades, flow grades, frame count, and percutaneous coronary intervention to long-term outcomes after thrombolytic administration in acute myocardial infarction. Circulation, Apr., 105 (16): 1909-13, 2002.

6- HEAD S.J., FAROOQ V., SERRUYS P.W. and KAPPETEIN A.P.: The SYNTAX score and its clinical implications. Heart, 100 (2): 169-77, 2014.

7- KOTZ S.: Concordance Correlation Coefficients. In: Encyclopedia of Statistical Sciences, 2005.

8- MEMBERS A.F., STEG P.G., JAMES S.K., ATAR D., BADANO L.P., LUNDQVIST C.B., et al.: 2012 ESC Guidelines for the management of acute myocardial infarction in patients presenting with ST-segment elevation. Eur. Heart J., 33 (20): 2569-619, 2012.

9- JERNBERG T., JOHANSON P., HELD C., SVENNBLAD B., LINDBÄCK J. and WALLENTIN L.: Association between adoption of evidence-based treatment and survival for patients with ST-elevation myocardial infarction. JAMA, 305 (16): 1677-84, 2011.

10- REGITZ-ZAGROSEK V., OERTELT-PRIGIONE S., PRESCOTT E., FRANCONI F., GERDTS E., FORYSTLUDWIG A., et al.: Gender in cardiovascular diseases: Impact on clinical manifestations, management, and outcomes. Eur. Heart J., 37 (1): 24-34, 2016.

11- BRIEGER D., EAGLE K., GOODMAN S., STEG G., BUDAJ A., WHITE K., et al.: Acute Coronary Syndromes Without Chest Pain, An Underdiagnosed and Undertreated High-Risk Group. Chest, 126 (2): 461-9, 2004.

12- KAUL P., ARMSTRONG P.W., SOOKRAM S., LEUNG B.K., BRASS N. and WELSH R.C.: Temporal trends in patient and treatment delay among men and women presenting with ST-elevation myocardial infarction. Am. Heart J., 161 (1): 91-7, 2011.

13- SHARIF D., ABU-SALEM M., SHARIF-RASSLAN A. and ROSENSCHEIN U.: Platelet counts on admission affect coronary flow, myocardial perfusion and left ventricular systolic function after primary percutaneous coronary intervention. Eur. Hear J. Acute Cardiovasc. Care, 2016.
14- KAPLAN S., KAPLAN S.T., KIRIS A. and GEDIKLI O.: Impact of initial platelet count on baseline angiographic finding and end-points in ST-elevation myocardial infarction referred for primary percutaneous coronary intervention. Int. J. Clin. Exp. Med., 7 (4): 1064-70, 2014.

15-ACET H., ERTAS F., AKIL M.A., OZYURTLU F., YILDIZ A., POLAT N., et al.: Novel predictors of infarctrelated artery patency for ST-segment elevation myocardial infarction: Platelet-to-lymphocyte ratio, uric acid, and neutrophil-to-lymphocyte ratio. Anatol. J. Cardiol., 15 (8): 648-56, 2015.

16- SAHIN D.Y., GÜR M., ELBASAN Z., Y1LD1Z A., KAYA Z., IÇEN Y.K., et al.: Predictors of preinterventional patency of infarct-related artery in patients with STsegment elevation myocardial infarction: Importance of neutrophil to lymphocyte ratio and uric acid level. Exp. Clin. Cardiol., 18 (2): e77-81, 2013.

17- SCHAAF M.J., MEWTON N., RIOUFOL G., ANGOULVANT D., CAYLA G., DELARCHE N., et al.: Pre-PCI angiographic TIMI flow in the culprit coronary artery influences infarct size and microvascular obstruction in STEMI patients. J. Cardiol., 67 (3): 248-53, 2016.

18- WANG R., MEI B., LIAO X., LU X., YAN L., LIN M., et al.: Determination of risk factors affecting the inhospital prognosis of patients with acute ST segment elevation myocardial infarction after percutaneous coronary intervention. BMC Cardiovasc. Disord., Sep. 12 (17): 243, 2017.

19- BAHREHMAND M., SADEGHI E., SHAFIEE A. and NOZARI Y.: Predictors of delayed and no-reflow as recognized with Thrombolysis in Myocardial Infarction [TIMI] flow grade following Primary Percutaneous Coronary Angioplasty. J. Med. Life. Mar. 14; 8 (Spec. Iss. 3): 59-65, 2015

20- SABIA P., AFROOKTEH A., TOUCHSTONE D.A., KELLER M.W., ESQUIVEL L. and KAUL S.: Value of regional wall motion abnormality in the emergency room diagnosis of acute myocardial infarction. A prospective study using two-dimensional echocardiography. Circulation, 1991.

21- KURTUL A., MURAT S.N., YARLIOGLUES M., DURAN M., ERGUN G., ACIKGOZ S.K., et al.: Association of platelet-to-lymphocyte ratio with severity and complexity of coronary artery disease in patients with acute coronary syndromes. Am. J. Cardiol., 2014. 


\section{تآثير العد الآولى للصفائح الدموية على نتائج قسطرة الشرايين التاجية

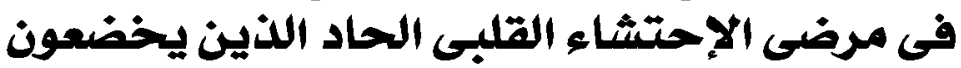 \\ للقسطرة القلبية العلاجية الآجية الآولية}

آشملت الدراسة مائة وخمسين مريضا آصيبوا بإحتشاء عضلة القلب الحاد وتمت المعالجة عن طريق القسطرة العلاجية الآولية على الشرايين

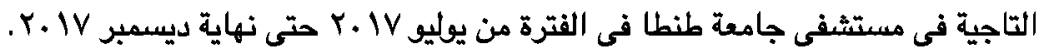

ققد كان الهدف من هذه الدراسة هو دراسة العلاقة بين العدد الآولى للصفائع الدموية بالدم ونتائج القسطرة القلية فى المرضى المصابين بالإحتشاء القلبى الصاد الذين يخضعون للقسطرة العلاجية الآولية المثرايين التاجية.

$$
\begin{aligned}
& \text { تم تقسيم حالات الدراسة إلى مجموعثين تبعا لدرجة دفق الثريان بمقياس TIMI إلى: } \\
& \text { • المجموعة الآولى: والتى كانت درجة دفق الثريان بمقياس TIMI هى صفر. } \\
& \text { • المجموعة الثانية: والتى كانت درجة دفق الثريان بمقياس TIMI هى من إلى إلى بـ }
\end{aligned}
$$

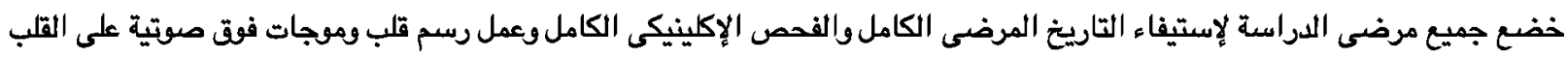

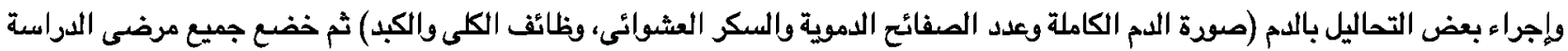
إلى القسطرة القليية العلاجية الطارئة.

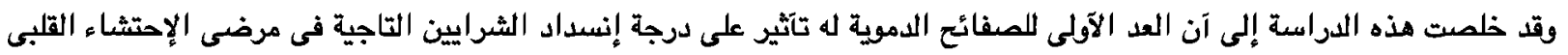

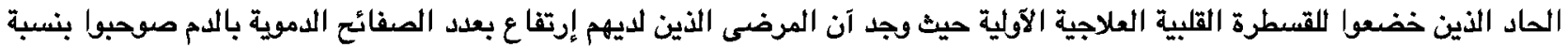

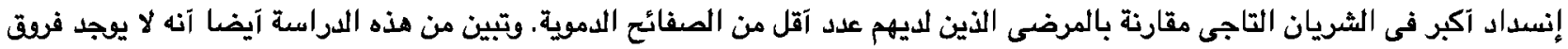

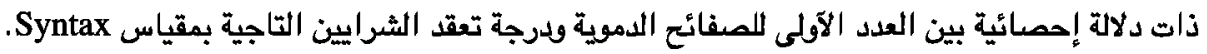

